\title{
Autism
}

The International Joumal of Research and Practice

\section{Gender Differences in Self-Reported Camouflaging in Autistic and Non-Autistic Adults}

\begin{tabular}{|c|c|}
\hline Journal: & Autism \\
\hline Manuscript ID & AUT-19-0085.R2 \\
\hline Manuscript Type: & Original Article \\
\hline Keywords: & $\begin{array}{l}\text { Camouflaging, Autism, Masking, Compensation, Gender Differences, } \\
\text { Females, Adult }\end{array}$ \\
\hline Abstract: & 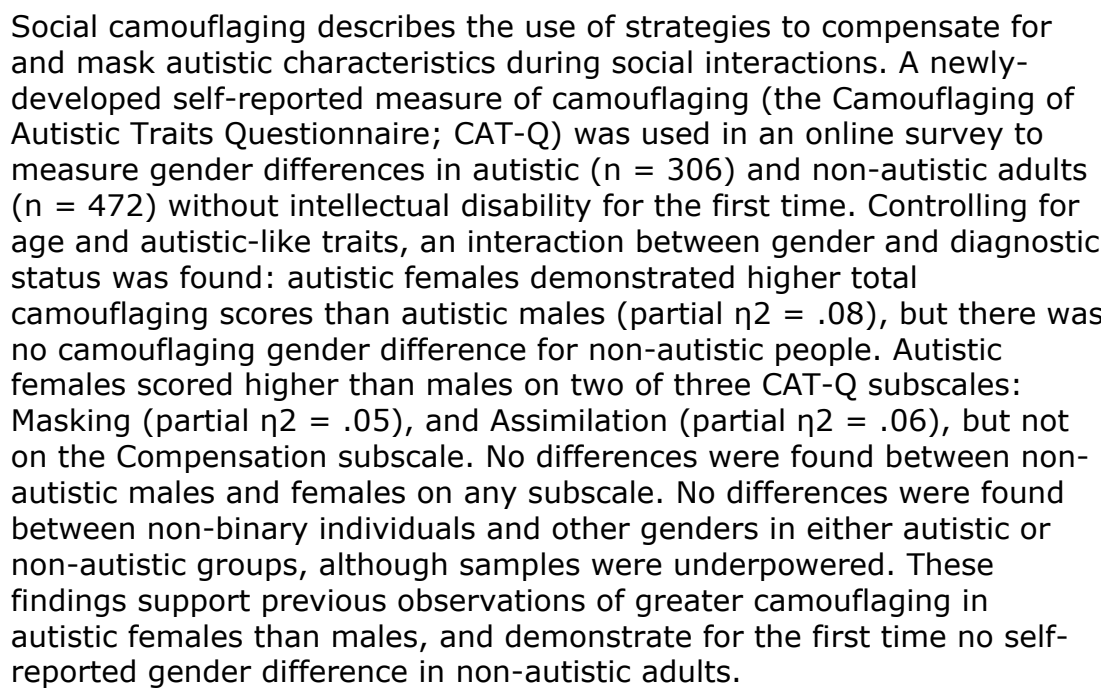 \\
\hline
\end{tabular}

\section{SCHOLARONE" Manuscripts}




\title{
Gender Differences in Self-Reported Camouflaging in Autistic and Non-
}

\author{
Autistic Adults \\ Laura Hull ${ }^{\text {a }}$, Meng-Chuan Lai ${ }^{\text {c d }}$, Simon Baron-Cohen ${ }^{c}$, Carrie Allison ${ }^{c}$, Paula Smith ${ }^{c}$, K V \\ Petrides ${ }^{\mathrm{e}} \&$ William Mandy ${ }^{\mathrm{a}}$ \\ a Research Department of Clinical, Educational \& Health Psychology, University College \\ London, London, UK \\ ${ }^{\mathrm{b}}$ Centre for Addiction and Mental Health and The Hospital for Sick Children, Department of \\ Psychiatry, University of Toronto, Toronto, Ontario, Canada \\ c Autism Research Centre, Department of Psychiatry, University of Cambridge, Cambridge, \\ UK \\ d Department of Psychiatry, National Taiwan University Hospital and College of Medicine, \\ Taipei, Taiwan \\ e London Psychometric Laboratory, University College London, London, UK \\ Corresponding Author \\ Laura Hull \\ Department of Psychology, University College London, 26 Bedford Way, London, United \\ Kingdom, WC1H 0AP \\ laura.hull.14@ucl.ac.uk \\ +4402076795365
}




\section{Acknowledgements}

The authors would like to thank Maya Bowri, Emogen Campbell, Andrew Dunlop and Lily Levy for assistance with data collection. A selection of these findings were presented at INSAR, May 2018 in Rotterdam, The Netherlands.

\section{Ethical approval}

All procedures performed in studies involving human participants were in accordance with the ethical standards of the institutional and/or national research committee and with the 1964 Helsinki declaration and its later amendments or comparable ethical standards.

\section{Funding}

Meng-Chuan Lai is supported by the O'Brien Scholars Program within the Child and Youth Mental Health Collaborative at the Centre for Addiction and Mental Health (CAMH) and The Hospital for Sick Children, Toronto, the Academic Scholars Award from the Department of Psychiatry, University of Toronto, the Slaight Family Child and Youth Mental Health Innovation Fund and The Catherine and Maxwell Meighen Foundation (both via CAMH Foundation), the Ontario Brain Institute via the Province of Ontario Neurodevelopmental Disorders (POND) Network, Women's Xchange, and the University of Toronto Department of Psychiatry Excellence Fund.

The authors received no financial support for the research, authorship, and/or publication of this article. 


\begin{abstract}
Social camouflaging describes the use of strategies to compensate for and mask autistic characteristics during social interactions. A newly-developed self-reported measure of camouflaging (the Camouflaging of Autistic Traits Questionnaire; CAT-Q) was used in an online survey to measure gender differences in autistic $(n=306)$ and non-autistic adults $(n=$ 472) without intellectual disability for the first time. Controlling for age and autistic-like traits, an interaction between gender and diagnostic status was found: autistic females demonstrated higher total camouflaging scores than autistic males (partial $\eta^{2}=.08$ ), but there was no camouflaging gender difference for non-autistic people. Autistic females scored higher than males on two of three CAT-Q subscales: Masking (partial $\eta^{2}=.05$ ), and Assimilation (partial $\eta^{2}=.06$ ), but not on the Compensation subscale. No differences were found between non-autistic males and females on any subscale. No differences were found between non-binary individuals and other genders in either autistic or non-autistic groups, although samples were underpowered. These findings support previous observations of greater camouflaging in autistic females than males, and demonstrate for the first time no self-reported gender difference in non-autistic adults.
\end{abstract}

\title{
Keywords
}

Autism, camouflaging, masking, compensation, gender differences, females, adult 
Social camouflaging is defined as the use of strategies to present a less visibly autistic persona to others during a social interaction (Hull et al., 2017; Lai et al., 2011).

Camouflaging behaviours may be conscious or unconscious, and specific strategies as well as their impact vary across individuals. Behaviours used to camouflage may also be a consequence of the development of cognitive compensatory strategies (Livingston \& Happé, 2017). Some common strategies include compensation for inherent autistic social differences, such as consciously learning 'scripts' for regular social interactions, or implicitly developing techniques to mask the appearance of autism, such as using speech patterns or sounds more often shown by non-autistic people (Hull et al., 2017; Parish-Morris et al., 2017). Camouflaging strategies are performed with the aim of reducing discrimination, smoothing social interactions, and achieving success in employment or education (Hull et al., 2017), although it still unknown to what extent these aims are achieved. Frequently-reported consequences of camouflaging are exhaustion, loss of identity, and mental health difficulties including suicidal thoughts (Bargiela, Steward, \& Mandy, 2016; Cassidy, Bradley, Shaw, \& Baron-Cohen, 2018; Tierney, Burns, \& Kilbey, 2016).

It has been suggested that females may experience and express their autism in different ways to males, resulting in under-diagnosis of females; this is known as the 'female autism phenotype' (Dworzynski, Ronald, Bolton, \& Happé, 2012; Gould \& Ashton-Smith, 2011; Lai, Lombardo, Auyeung, Chakrabarti, \& Baron-Cohen, 2015). The proposed female phenotype may represent differences in quantity of behaviours (such as higher levels of internalising disorders such as depression or anxiety; Lai et al., 2015) or in quality of behaviours (such as differences in friendship experiences; Sedgewick, Hill, Yates, Pickering, \& Pellicano, 2015) between males and females at group levels, with significant individual variation within each gender. 
Greater camouflaging of autistic characteristics, especially during clinical assessments or outside of the home, has been proposed as one way in which autistic girls and women may be missed by assessments that focus on 'typical' autism behaviours (Kopp \& Gillberg, 1992). There may be many factors contributing to this gender-variant presentation. Biological or developmental differences between genders may lead to greater cognitive abilities related to camouflaging (Lehnhardt et al., 2013); while females may also be held to higher standards of social skills (Hull et al., 2017). Autistic females may also experience greater stigmatisation for appearing autistic than males, and this may relate to the intersection of multiple marginalised identities which create unique pressures for people who are both autistic and female, compared to either group separately (Cage \& Troxell-Whitman, 2019). All these factors may interact to produce greater expectation, necessity, and ability to camouflage autism for females compared to males.

If gender differences in camouflaging in the hypothesised direction are found in autistic individuals, but not in non-autistic individuals, this supports the role of camouflaging in the female autism phenotype. In contrast, if similar gender differences are found in non-autistic males and females, this suggests that gender differences in camouflaging may reflect general social expectations for females to be more sociable and amenable than males (Kreiser \& White, 2014). The present study is the first known to test these hypotheses by comparing selfreported camouflaging behaviours in a large sample of autistic and non-autistic adults. There has also been limited research into the extent of camouflaging in individuals who do not identify as either male or female; this study seeks to address this by including non-binary individuals in analyses of gender differences.

One approach to measuring camouflaging focuses on the 'discrepancy' between underlying social and cognitive abilities, and behavioural expression of autism/social abilities (Lai et al., 2017). Using this approach, camouflaging can only be measured in those identified as having 
difficulties in the areas of interest compared to the standards expected of neurotypical individuals, since non-autistic individuals would theoretically have minimal discrepancy scores (although see Livingston, Colvert, Bolton, \& Happé, 2018, for evidence of compensation for mentalizing difficulties in non-autistic individuals). Autistic women show a greater discrepancy between internal autistic status and external behavioural presentation than autistic men (Lai et al., 2017), and the level of which is associated with neural activation related to self-referential cognition in autistic women specifically (Lai et al., 2018). Behavioural assessment of camouflaging suggests that autistic girls use more linguistic strategies to camouflage their social communication difficulties than boys (Boorse et al., 2019; Parish-Morris et al., 2017). Autistic girls also tend to have higher parent-observed adaptive difficulties and autistic characteristics when they present comparable levels of clinician-rated autistic behaviours compared to autistic boys (Ratto et al., 2018).

An alternative approach which aims to directly identify and measure camouflaging strategies allows for comparison between groups regardless of diagnostic status or perceived difficulties (Hull et al., 2018). Following this 'observational/reflective' approach, Dean and colleagues (2017) observed suggestive evidence of camouflaging in certain environments seen in autistic girls, but not in autistic boys or non-autistic children. This suggests that gender differences in camouflaging may be unique to autistic individuals, and that non-autistic males and females do not differ in the extent to which they display any camouflaging behaviours. However, this study focused only on a small number of children in a playground setting; these findings need to be tested in larger samples across broader age ranges. In a study of adults, autistic men and women were equally likely to spontaneously report using camouflaging strategies (Cage, Di Monaco, \& Newell, 2017).

Differences in camouflaging levels across genders may lead to differences in risk of mental health problems and reduced wellbeing (Lai et al., 2017); it is therefore important to assess 
how much autistic individuals of different genders are camouflaging in everyday life, and compare this to camouflaging in non-autistic individuals. Camouflaging, like autistic traits, likely exists on a continuum across the entire population. However, individuals with higher levels of autistic characteristics are also likely to camouflage these more (Hull et al., 2018), and so higher levels of camouflaging in one gender may simply reflect more autistic characteristics to camouflage. As a supplementary analysis, and to thoroughly test the hypothesis that autistic females camouflage more than males because of differences in social expectation and/or behavioural presentation (Cage \& Troxell-Whitman, 2019; Lai et al., 2011), it is also important to control for autistic traits, separately to assessing the extent of real-life camouflaging across genders. There may be age-related differences in the extent to which individuals camouflage, based on social demands, experience, mental health, and other factors (Cage \& Troxell-Whitman, 2019; Hull et al., 2017), and so age should be included in comparisons of camouflaging across groups and genders.

A new self-report measure of camouflaging, the Camouflaging Autistic Traits Questionnaire (CAT-Q; Hull et al., 2018), has been developed based on autistic adults' lived experiences of camouflaging to provide a measure of self-recognised intentions to camouflage, regardless of behavioural outcome. The CAT-Q uses an observational/reflective approach to measuring camouflaging. Items describing camouflaging strategies were developed from autistic adults' self-reported camouflaging behaviours, and thus reflect behaviours experienced by autistic individuals themselves, which may not all be identified through external observation. Unlike other measures of camouflaging, the CAT-Q has demonstrated equivalency of psychometric structure in males and females with and without an autism diagnosis (Hull et al., 2018), and, therefore, can be used to compare camouflaging behaviours between these groups. 
The present study had two key aims. First, this was the first study to test gender differences in self-reported camouflaging behaviours in both autistic and non-autistic adults, including people of non-binary gender. We hypothesised that autistic females would camouflage more than autistic males, following the predictions of the female autism phenotype hypothesis and in support of some previous research. Gender differences for non-binary autistic individuals' camouflaging, or that of any non-autistic participants, were not hypothesised as there has been no prior research examining this.

Second, we also aimed to examine whether gender differences in camouflaging of autistic traits reflect underlying levels of autistic traits, and whether this is comparable across diagnostic groups. Autistic traits were added as a covariate in supplementary analyses, to determine whether any gender differences in real-life identified in autistic or non-autistic groups remained once variation in autistic traits between genders was controlled for.

\section{Methods}

\section{Participants}

Autistic and non-autistic participants were recruited through social media, through the [removed for review] database, and through word-of-mouth. Participants self-reported an official autism diagnosis from a qualified healthcare professional and were asked to detail the label of diagnosis (e.g. Autism, Asperger's Syndrome, Autism Spectrum Disorder), the age they were diagnosed, and the type of healthcare professional who diagnosed them. Those who reported being self-diagnosed were automatically excluded from the study and did not complete any further questions. Gender was measured by asking participants to report the gender they identified as (male, female or 'other gender'). Characteristics of the sample and mean scores on all variables are included in Table 1. 
[INSERT TABLE 1 HERE]

Power analysis using $\mathrm{G}^{*}$ Power (Faul, Erdfelder, Lang, \& Buchner, 2007) determined that a minimum sample of 54 per group would be necessary to detect medium-sized differences (partial $\eta^{2}=0.5$ ) in an ANCOVA between autistic and non-autistic males, females, and nonbinary individuals' camouflaging score while controlling for age and autistic-like traits. Individuals who identified as non-binary (neither male nor female; $\mathrm{N}=43 ; 16$ with autism, 27 without autism) were included in analyses, but results are presented and discussed as preliminary only, as this sample was not well-powered enough to detect the predicted effect sizes. The final sample included 778 participants in total (see Table 1). An additional 53 individuals did not report their gender, and were not included in any analyses.

\section{Measures}

\section{Camouflaging of Autistic Traits Questionnaire (CAT-Q; Hull et al., 2018).}

This is a 25 -item self-report questionnaire measuring strategies used to camouflage autistic traits and comprising three factors (Compensation, Masking, and Assimilation) which are summed up to produce a total score from 25 to 175 , with higher scores representing greater levels of camouflaging. The CAT-Q has been validated in autistic and non-autistic male and female samples, and has demonstrated measurement equivalence across gender and diagnostic groups (Hull et al., 2018). Internal consistency in this sample was high $(\alpha=0.94)$.

\section{Broad Autism Phenotype Questionnaire (BAPQ; Hurley, Losh, Parlier, Reznick, \& Piven, 2007).}

This is a 36-item self-report measure of traits associated with the broader autism phenotype (BAP). BAP characteristics are associated with greater genetic liability for autism, and are found across the population and at especially high levels in relatives of those with an autism diagnosis. Although it was designed as a measure of autistic-like traits in relatives of autistic 
people, the BAPQ has been demonstrated to be a good measure of autistic-like traits across the general population, including in autistic individuals (Nishiyama et al., 2014). The BAPQ has good sensitivity (Sasson et al., 2013) and specificity (Hurley et al., 2007) for the broader autism phenotype. Internal consistency in this sample was high $(\alpha=0.96)$. Minimum score is 0 , maximum score is 6 when scores are averaged across the total questionnaire.

\section{Procedure}

Participants followed an online link to the study, hosted by Qualtrics, where they read the information sheet and completed an informed consent form. They completed demographic questions and then the CAT-Q and BAPQ as part of a broader battery of questionnaires, online.

Ethical approval for this study was obtained from [removed for review] Research Ethics Committee (ID numbers [removed for review]). Informed consent was obtained from all individual participants in this study.

\section{Analysis}

All analyses were performed in R (R Core Team, 2013) and SPSS Version 23 (IBM Corp., 2015).

The following analyses of covariance were performed to test the effect of gender and diagnostic group, and the interaction between gender and diagnostic group, on CAT-Q total and on each of its three subscales (Compensation, Masking, and Assimilation). Initial analyses were performed in the total sample, with follow-up analyses performed in the autistic and non-autistic samples, and in male, female, and non-binary subsamples, separately. Bonferroni corrections were used for all follow-up analyses to account for multiple comparisons, with a corrected significance value of $\alpha=.005$. Partial eta squared was used as a measure of effect size for MANCOVA and ANCOVA (where values under .04 = 
small effect, values of $.04-.10=$ moderate effect, and values above $.14=$ large effect), and Cohen's d used as a measure of effect size for planned comparison tests (where values under $0.4=$ small effect, values of $0.5-0.7=$ moderate effect, and values above $0.8=$ large effect) Both effect sizes were interpreted following Cohen (1988; reported in Lenhard \& Lenhard, 2016).

Firstly, main and interaction effects of gender and diagnostic group were examined while controlling for participant's age, through analysis of covariance (ANCOVA) for Total CATQ and MANCOVA for CAT-Q subscales. Univariate ANCOVAs were run separately in autistic and non-autistic groups to examine main effects of gender (with planned comparisons between each of the three genders when significant main effects were found), and in each gender to examine main effects of diagnostic group. These analyses aimed to identify the real-life levels of camouflaging across genders and groups.

Secondly, all analyses were repeated while controlling for participants' age and autistic traits (BAPQ total score). These analyses aimed to identify whether gender and group differences in camouflaging simply reflect differences in the amount of autistic characteristics that need to be camouflaged, or are a result of other factors.

\section{Results}

Two-way ANOVA comparing age revealed main effects of diagnostic group (autistic participants were significantly older than non-autistic participants $(F[1,785]=52.40, \mathrm{p}<$ .001 , partial $\left.\eta^{2}=.06\right)$, gender $(F[2,785]=12.02, p<.001)$, and an interaction between diagnostic group and gender $(\mathrm{F}[2,785]=4.64, \mathrm{p}=.01)$. Follow-up comparisons indicated that in the autistic sample, males were older than females $(p<.001)$ and non-binary individuals $(p$ $<.001)$; there was no difference between females' and non-binary participants' ages. In the non-autistic sample, there were no gender differences in age. Autistic participants (mean 
BAPQ score $=4.30, \mathrm{SD}=0.71)$ had significantly higher levels of autistic-like traits than nonautistic participants (mean BAPQ score $=3.18, \mathrm{SD}=0.74 ; \mathrm{t}(777)=20.55, \mathrm{p}<.001$ ).

Gender distributions on unadjusted Total CAT-Q scores in autistic and non-autistic samples are presented in Figure 1. Distributions on the CAT-Q subscales are presented in Supplementary Figures 1a-c. 'Density' represents the proportion of participants in each group who scored at each level of the CAT-Q or its subscales; distributions for non-autistic samples have been reflected to allow group comparison, and still represent positive values.

\section{[INSERT FIGURE 1 HERE]}

\section{Gender and Group differences with Age as a covariate}

Figure 2 shows interactions between gender and diagnostic group for total and subscale CATQ scores, when age was included as a covariate. See Table 1 for unadjusted means for all variables.

\section{[INSERT FIGURE 2 HERE]}

Total CAT-Q score

The first ANCOVA revealed no main effect of gender $(\mathrm{F}[3,781]=0.23, \mathrm{p}=.63)$, a significant main effect of diagnostic group, with autistic participants scoring higher than non-autistic participants $(\mathrm{F}[3,781]=232.24, \mathrm{p}<.001)$, and an interaction between gender and diagnostic group for Total CAT-Q score $(\mathrm{F}[3,781]=26.27, \mathrm{p}<.001)$. The assumption of equality of error variance was met for this model.

In order to further explore this interaction, follow-up ANCOVA in the autistic sample revealed a main effect of gender $\left(F[2,306]=9.67, p<.001\right.$, partial $\left.\eta^{2}=.06\right)$, with pairwise comparisons indicating autistic females scored higher than males $(\mathrm{p}<.001, \mathrm{~d}=.65)$, and no difference between non-binary individuals and males or females. In the non-autistic sample, 
follow-up ANCOVA demonstrated a main effect of gender $(\mathrm{F}[2,478]=8.98, \mathrm{p}<.001$, partial $\left.\eta^{2}=.04\right)$, with pairwise comparisons indicating non-autistic non-binary individuals scored higher than females $(\mathrm{p}<.001, \mathrm{~d}=.73)$ but not males. Non-autistic males did not score differently to any other gender at the corrected alpha level.

Follow-up ANCOVAs were performed to examine diagnostic group differences separately for each gender. Autistic females scored higher than non-autistic females $(F[1,437]=218.95$, $\mathrm{p}<.001$, partial $\left.\eta^{2}=.34\right)$. Autistic males scored higher than non-autistic males $(\mathrm{F}[1,300]=$ $29.16, p<.001$, partial $\eta^{2}=.09$ ). No difference was found between autistic and non-autistic non-binary participants $\left(\mathrm{F}[1,46]=2.96, \mathrm{p}=.09\right.$, partial $\left.\eta^{2}=.06\right)$.

\section{CAT-Q Subscales}

Overall for the three subscales, MANCOVA revealed no main effect of gender $(\mathrm{F}[3,781]=$ $1.70, p=.19)$, a main effect of diagnostic group with autistic participants scoring higher than non-autistic participants $(\mathrm{F}[3,781]=241.12, \mathrm{p}<.001)$, and an interaction between gender and diagnostic group $(\mathrm{F}[3,781]=15.71, \mathrm{p}<.001)$. The assumption of equality of covariance matrices was not met for this model, therefore Pillai's Trace was used as a robust multivariate statistic.

Follow-up ANCOVA in the autistic sample revealed a significant multivariate effect of gender (Pillai's Trace $=.02, \mathrm{~F}[6,604]=4.23, \mathrm{p}<.001$, partial $\eta^{2}=.04$ ), and univariate effects of gender in the Masking $\left(F[2,306]=6.29, p=.002\right.$, partial $\left.\eta^{2}=.04\right)$ and Assimilation $\left(F[2,306]=783.25, p<.001\right.$, partial $\left.\eta^{2}=.06\right)$ subscales. No main effect of gender was found for the Compensation subscale when adjusting for the corrected alpha (F[2, $306]=4.07, p=.02$, partial $\eta^{2}=.03$ ). Pairwise comparisons were conducted to assess gender differences in the Masking and Assimilation subscales. Autistic females scored higher than 
autistic males on the Masking subscale $(\mathrm{p}=.001, \mathrm{~d}=.43)$ and the Assimilation subscale $(\mathrm{p}<$ $.001, \mathrm{~d}=.51)$. No other differences were significant at the corrected alpha level.

In the non-autistic sample, follow-up ANCOVA demonstrated a significant multivariate effect of gender (Pillai's Trace $=0.44, F[6,948]=3.52, p=.002$, partial $\eta^{2}=.02$ ), and univariate effect of gender for the Compensation subscale only $(F[2,478]=9.21, \mathrm{p}<.001$, partial $\left.\eta^{2}=.04\right)$. Pairwise comparisons revealed that non-autistic males scored higher than females $(\mathrm{p}=.005, \mathrm{~d}=.23)$, and that non-binary participants scored higher than females $(\mathrm{p}<$ $.001, \mathrm{~d}=.72)$ on Compensation.

Follow-up ANCOVAs were conducted to examine the effect of diagnostic group separately in each gender. A main effect of diagnostic group was found in females (Pillai's Trace $=.43$, $\mathrm{F}[3,433]=108.60, \mathrm{p}<.001$, partial $\left.\eta^{2}=.43\right)$, with autistic females scoring higher than nonautistic females on Compensation $\left(F[1,437]=212.28, p<.001\right.$, partial $\left.\eta^{2}=.33\right)$, Masking $\left(\mathrm{F}[1,437]=24.47, \mathrm{p}<.001\right.$, partial $\left.\eta^{2}=.05\right)$, and Assimilation subscales $(\mathrm{F}[1,437]=$ $262.38, \mathrm{p}<.001$, partial $\eta^{2}=.38$ ). A main effect of diagnostic group was also found in males (Pillai's Trace $=.25, \mathrm{~F}[3,296]=32.82, \mathrm{p}<.001$, partial $\left.\eta^{2}=.25\right)$, and autistic males scored higher than non-autistic males on the Compensation $\left(F[1,300]=37.89, p<.001\right.$, partial $\eta^{2}=$ $.11)$ and Assimilation subscales $\left(F[1,300]=52.06, p<.001\right.$, partial $\left.\eta^{2}=.15\right)$. In non-binary participants, no main effect of diagnostic group was found at the corrected significance level (Pillai's Trace $=.23, \mathrm{~F}[3,42]=4.20, \mathrm{p}=.01$, partial $\eta^{2}=.23$ ).

\section{Gender differences with Age and Autistic Traits as covariates}

Total CAT-Q score

The first ANCOVA revealed no main effect of gender $(\mathrm{F}[3,737]=0.01, \mathrm{p}=.99)$, a significant main effect of diagnostic group with autistic participants scoring higher than non-autistic participants $(\mathrm{F}[3,737]=11.25, \mathrm{p}<.001)$, and an interaction between gender and diagnostic 
group for Total CAT-Q score $(\mathrm{F}[3,737]=12.87, \mathrm{p}<.001)$. The assumption of equality of error variance was met for this model.

Figure 3 shows interactions between gender and diagnostic group for total and subscale CATQ scores, when age and autistic-like traits were included as covariates.

\section{[INSERT FIGURE 3 HERE]}

Follow-up ANCOVA in the autistic sample revealed a main effect of gender $(\mathrm{F}[2,299]=$ $6.98, p=.001$, partial $\left.\eta^{2}=.05\right)$, with pairwise comparisons indicating autistic females scored higher than males $(\mathrm{p}<.001, \mathrm{~d}=.47)$, and no difference between non-binary individuals and males or females. In the non-autistic sample, follow-up ANCOVA demonstrated no main effect of gender $\left(F[2,442]=2.61, p=.07\right.$, partial $\left.\eta^{2}=.01\right)$.

Follow-up ANCOVAs were performed to examine diagnostic group differences separately for each gender. Autistic females scored higher than non-autistic females $(F[1,415]=14.98$, $\mathrm{p}<.001$, partial $\left.\eta^{2}=.04\right)$. No difference was found between autistic and non-autistic males $(F[1,283]=0.12, p=.73)$ or non-binary participants $(F[1,39]=0.20, p=.65)$.

\section{CAT-Q Subscales}

Overall for the three subscales, MANCOVA revealed no main effect of gender $(\mathrm{F}[3,735]=$ $2.23, \mathrm{p}=.08$ ), a main effect of diagnostic group with autistic participants scoring higher than non-autistic participants $(\mathrm{F}[3,735]=36.00, \mathrm{p}<.001)$, and an interaction between gender and diagnostic group $(F[3,735]=7.70, p<.001)$. The assumption of equality of covariance matrices was not met for this model, therefore Pillai's Trace was used as a robust multivariate statistic.

Follow-up ANCOVA in the autistic sample revealed a significant multivariate effect of gender (Pillai's Trace $=.08, \mathrm{~F}[6,588]=3.81, \mathrm{p}=.001$, partial $\left.\eta^{2}=.04\right)$, and univariate 
effects of gender in the Masking $\left(F[2,299]=6.20, p=.002\right.$, partial $\left.\eta^{2}=.04\right)$ and Assimilation $\left(\mathrm{F}[2,299]=7.50, \mathrm{p}=.001\right.$, partial $\left.\eta^{2}=.05\right)$ subscales. Pairwise comparisons were conducted to assess gender differences in the Masking and Assimilation subscales. Autistic females scored higher than autistic males on the Masking subscale $(p=.001, d=.44)$ and the Assimilation subscale $(\mathrm{p}<.001, \mathrm{~d}=.40)$. No other differences were significant at the corrected alpha level.

In the non-autistic sample, follow-up ANCOVA demonstrated no significant multivariate effect of gender (Pillai's Trace $=0.25, \mathrm{~F}[6,874]=1.86, \mathrm{p}=.08$, partial $\eta^{2}=.01$ ) and so further analyses were not performed.

Follow-up ANCOVAs were conducted to examine the effect of diagnostic group separately in each gender. A main effect of diagnostic group was found in females (Pillai's Trace $=.07$, $F[3,410]=9.99, p<.001$, partial $\eta^{2}=.07$, with autistic females scoring higher than nonautistic females on Compensation $\left(\mathrm{F}[1,415]=28.59, \mathrm{p}<.001\right.$, partial $\left.\eta^{2}=.07\right)$ only. A main effect of diagnostic group was also found in males (Pillai's Trace $=.07, \mathrm{~F}[3,278]=6.99, \mathrm{p}<$ .001 , partial $\eta^{2}=.07$ ), but diagnostic group differences on each subscale were not significant at the corrected alpha level. In non-binary participants, no main effect of diagnostic group was found (Pillai's Trace $=.18, F[3,37]=2.66, p=.06$, partial $\eta^{2}=.18$ ).

\section{Discussion}

This was the first study to compare self-reported camouflaging behaviours between autistic and non-autistic men, women, and non-binary people.

A consistent finding was that autistic females had higher camouflaging scores than autistic males. Effect sizes were moderate, with the largest differences found for Total CAT-Q. This supports our hypothesis that autistic females camouflage more than males, and suggests that autistic women may use more masking strategies, and experience greater pressure than men 
to adapt their behaviours in order to assimilate with others. No gender difference was found on the Compensation subscale, suggesting autistic individuals of all genders may use compensatory strategies to a similar extent.

Non-binary autistic people had higher total CAT-Q scores than females when controlling for age only, suggesting that these individuals may be at particular risk of the negative outcomes associated with camouflaging. However, this difference was not found when autistic traits were controlled for, suggesting that their higher levels of camouflaging may arise because they have more autistic traits to camouflage than females. However, the number of autistic non-binary participants in this sample was very small, and so the analyses were likely underpowered to detected small group differences.

In contrast, gender differences in non-autistic individuals were minimal, and were not maintained when autistic traits were controlled for. Non-autistic males reported slightly higher levels of camouflaging than non-autistic females, but this difference was not maintained when we controlled for levels of autistic traits. The implication is that, compared to non-autistic females, non-autistic males may use slightly more camouflaging, reflecting the fact that they have somewhat higher levels of autistic traits (Robinson et al., 2011). Nonbinary non-autistic individuals had higher Total CAT-Q and Compensation scores than females, which may be accounted for by their higher mean levels of autistic traits (see Table 1), as these differences were not found when controlling for autistic traits. Again, however, the sample size was underpowered to detect small differences between non-binary and other participants.

Consistent group differences were found between autistic and non-autistic females, which were maintained when controlling for autistic traits. Again, this suggests that there is an interaction between being female and being autistic which produces greater camouflaging 
than the simple additive effects of each separately. Interestingly, differences between autistic and non-autistic males were not maintained when autistic traits were controlled for, suggesting that males across diagnostic groups camouflage their autistic characteristics to a similar extent (but that autistic males have higher levels of autistic traits, and so use more camouflaging strategies in real life). No differences between autistic and non-autistic nonbinary participants were found, which is likely to reflect the small samples of each.

Overall, the pattern of gender differences suggests a relatively similar use of specific compensatory strategies in autistic males, females, and non-binary people (as measured by similar scores on the Compensation subscale), but greater use of specific Masking and Assimilation strategies by females. One explanation for this may be differences in the gendered experiences of autistic females and males, as a product of both cultural gender norms and being held to the standards of typically developing females and males respectively (Cage \& Troxell-Whitman, 2019). Kreiser and White (2014) describe an interaction between cultural, inter- and intra-personal, and biological factors affecting gender and individual development, which they suggest may produce variation in both innate autistic experience and external autistic presentation. Autistic females may perceive greater expectations to be acting similarly to typically developing peers than are felt by autistic males (Bargiela, Steward \& Mandy, 2016; Dean et al., 2014), and so may camouflage to a greater extent in order to try and fit in (Kreiser \& White, 2014; Tierney et al., 2016). This pattern of difference was not found in the non-autistic sample. This indicates that although the kind of intention and behavioural strategies measured by the CAT-Q are utilized across autistic and non-autistic adults, impact of gender (and theoretically, gendered contexts) is more evident and unique in the autistic compared to non-autistic population.

Participants in this study were mostly European or North American and in early/middle adulthood on average, and so mostly grew up in Westernised cultures during the 1970s, 80s, 
and 90s. Gender-based stereotypes and rigid gender binaries during childhood and adolescence may have contributed to this greater pressure to camouflage autistic characteristics for autistic females than males. It will be important for future research to understand why such impact is more obvious in the autistic than the neurotypical population (e.g., do autistic people of this age conform more to gender norms than their neurotypical peers?) and whether the same will be found in the younger generations, for whom conventional, binary gender norms are relaxing. It may be that the combined identities of being autistic and female result in greater stigmatisation of difference, which leads to more efforts to fit in for autistic women than other groups (Cage \& Troxell-Whitman, 2019). As such, it will be a task to look at autistic individuals of all genders who develop their (autistic) identities under the current, more fluid conceptions of gender and neurodiversity in many Westernised cultures (although it remains to be seen as to whether gender equality in society impacts social conceptions of gender; MacPhee \& Prendergast, 2019; Prendergast \& MacPhee, 2018), and see whether the observed gender differences remain.

Gender differences in the autistic sample remained significant even when controlling for autistic-like traits, suggesting that higher levels of camouflaging in females are not due to having more autistic traits to camouflage than males, but due to greater extent of camouflaging of the autistic traits they do have, perhaps because of greater social expectations for females than males (Bargiela, Steward \& Mandy, 2016). Autistic women may experience unique and more extreme pressures than either autistic men or typically developing women, in part because of the intersection of their identities as neurodivergent and female (Cage \& Troxell-Whitman, 2019), which may lead to differences in behavioural expression of autistic characteristics compared to the 'typical' male presentation. This supports suggestions that camouflaging forms part of the female phenotype of autism (Head, 
McGillivray, \& Stokes, 2014; Kirkovski, Enticott, \& Fitzgerald, 2013; Lai et al., 2015; Lehnhardt et al., 2015).

Camouflaging of autistic traits may be more predominant in autistic females, and may partially account for the missed and later diagnosis of autism found for many females (Duvekot et al., 2017; Dworzynski et al., 2012; Shattuck et al., 2009). Previous research has also suggested that camouflaging may lead to mental health difficulties amongst autistic females, particularly anxiety and exhaustion related to the pressures of maintaining the 'façade' (Bargiela et al., 2016; Tierney et al., 2016), and suicidal thoughts (Cassidy et al., 2018).

Our findings contradict those by Cage and Troxell-Whitman (2019), who did not find difference between autistic males and females using the total CAT-Q score. These researchers did not control for autistic traits, therefore it is possible that male participants in their study had higher levels of autistic traits to camouflage than females, resulting in comparable overall camouflaging scores. Further research in a range of broader samples is necessary to determine the exact nature and size of any gender differences in camouflaging, using a range of methodologies. We would also suggest that, once the literature reaches a sufficient size, meta-analysis is the best way to produce a definitive answer on the direction and size of gender differences in camouflaging using a variety of samples and methods. However, autistic males in the current study camouflaged at significantly higher levels than non-autistic males, and at equivalent levels to autistic non-binary individuals, when controlling for age only. These findings support previous research arguing that camouflaging is not an exclusively female phenomena (Cage et al., 2017; Lai et al., 2017; Livingston et al., 2018). As demonstrated in the first set of analysis, controlling only for age, autistic males and non-binary individuals are also likely to experience the negative consequences associated with camouflaging, and there may even be greater impact on mental health for men than 
women, possibly due to reduced experience of camouflaging and other gender-related demands (Hull et al., 2017; Lai et al., 2017).

\section{Limitations and Strengths}

A significant limitation of this study is that only adults who were able to access and answer the online questionnaire were included in the study. While the online nature of this study enabled participation by individuals who prefer written to spoken language, there was no representation of autistic individuals who may be unable to reflect upon and express their behaviours through written English. Furthermore, the average age of autism diagnosis was 31.92 years, suggesting that these findings may be limited to individuals who are diagnosed in adulthood. These individuals may be more likely than others to camouflage their autism, having remained unidentified for so long. Therefore, it is still an open question as to whether the findings could be generalised to autistic people who were diagnosed earlier in life (e.g. in childhood).

In addition, participants' autistic status was based on self-reported disclosure, and was not independently verified. However, participants were also asked to give details of who gave them the diagnosis, and those who reported being diagnosed by someone other than a clinician or healthcare team were excluded from the study. More information about participants' diagnostic experiences, and other individual characteristics such as ethnicity, is important for future research to understand factors affecting variability in camouflaging.

Although we have reported all results with regards to non-binary participants, we emphasise that both autistic and non-autistic samples of non-binary individuals were underpowered to detect the expected effect sizes. This may account for the non-significance of most results regarding non-binary participants. We therefore interpret these results with caution, and suggest that further study with larger samples of non-binary individuals is conducted before 
any conclusions are drawn regarding non-binary camouflaging levels. Additionally, the CATQ has not been psychometrically validated in non-binary populations, therefore it may not be an appropriate measure to use with this group. However, as a relatively large proportion of autistic individuals identify as non-binary (Cooper, Smith, \& Russell, 2018; Dewinter, De Graaf \& Begeer, 2017), we believe these results are an important first step to learning more about the mechanisms and consequences of camouflaging across all genders.

Despite the aforementioned limitations, this study had multiple strengths. First, it is unique in that a psychometrically validated measure was used to assess and compare camouflaging behaviours between autistic and non-autistic males, females, and non-binary individuals. Second, it is based on the largest sample used to assess gender differences in camouflaging so far, strengthening previous findings of greater camouflaging in autistic females. Third, by controlling for age and autistic-like traits we demonstrate that self-reported camouflaging is not exclusively related to the development of typical social skills, and that gender differences in camouflaging exist for autistic individuals only.

The findings suggest that autistic women camouflage their autistic traits to a greater degree than autistic men, and therefore clinicians and other service providers should consider camouflaging when assessing women's autistic characteristics and their impact on daily functioning and wellbeing. However, autistic men and non-binary individuals also camouflage their autism at high levels, and should also be included in research looking at the consequences of camouflaging. Validated measures such as the CAT-Q may be used by individuals and in clinical settings to identify relevant camouflaging strategies and adapt behaviour as necessary to minimise negative outcomes.

\section{Acknowledgements}


The authors would like to thank Maya Bowri, Emogen Campbell, Andrew Dunlop and Lily Levy for assistance with data collection. A selection of these findings were presented at INSAR, May 2018 in Rotterdam, The Netherlands. 


\section{References}

Bargiela, S., Steward, R., \& Mandy, W. (2016). The experiences of late-diagnosed women with Autism Spectrum Conditions: An investigation of the female autism phenotype. Journal of Autism and Developmental Disorders, 46(10), 3281-3294. https://doi.org/10.1007/s10803-016-2872-8

Boorse, J., Cola, M., Plate, S., Yankowitz, L., Pandey, J., Schultz, R. T., \& Parish-morris, J. (2019). Linguistic markers of autism in girls : evidence of a " blended phenotype" during storytelling. Molecular Autism, 1-12.

Cage, E., Di Monaco, J., \& Newell, V. (2017). Experiences of autism acceptance and mental health in autistic adults. Journal of Autism and Developmental Disorders, 5-8.

Cage, E., \& Troxell-Whitman, Z. (2019). Understanding the Reasons, Contexts and Costs of Camouflaging for Autistic Adults. Journal of Autism and Developmental Disorders, O(0), 0. https://doi.org/10.1007/s10803-018-03878-x

Cassidy, S., Bradley, L., Shaw, R., \& Baron-Cohen, S. (2018). Risk markers for suicidality in autistic adults. Molecular Autism, 9(42), 1-14. https://doi.org/10.1186/s13229-0180226-4

Cohen, J. (1988). Set Correlation and Contingency Tables. Applied Psychological Measurement, 12(4), 425-434. https://doi.org/10.1177/014662168801200410

Cooper, K., Smith, L. G. E., \& Russell, A. J. (2018). Gender Identity in Autism : Sex Differences in Social Affiliation with Gender Groups. Journal of Autism and Developmental Disorders, O(0), 0. https://doi.org/10.1007/s10803-018-3590-1

Dean, M., Harwood, R., \& Kasari, C. (2017). The art of camouflage: Gender differences in the social behaviors of girls and boys with autism spectrum disorder. Autism, 21(6), 
678-689. https://doi.org/10.1177/1362361316671845

Dean, Michelle, Kasari, C., Shih, W., Frankel, F., Whitney, R., Landa, R., ... Harwood, R. (2014). The peer relationships of girls with ASD at school: comparison to boys and girls with and without ASD. Journal of Child Psychology and Psychiatry, and Allied Disciplines, 55(11), 1218-1225. https://doi.org/10.1111/jcpp.12242

Dworzynski, K., Ronald, A., Bolton, P., \& Happé, F. (2012). How different are girls and boys above and below the diagnostic threshold for autism spectrum disorders? Journal of the American Academy of Child and Adolescent Psychiatry, 51(8), 788-797. https://doi.org/10.1016/j.jaac.2012.05.018

Faul, F., Erdfelder, E., Lang, A.-G., \& Buchner, A. (2007). G*Power 3: A flexible statistical power analysis program for the social, behavioral, and biomedical sciences. Behavior Research Methods, 39, 175-191.

Gould, J., \& Ashton-Smith, J. (2011). Missed diagnosis or misdiagnosis? Girls and women on the autism spectrum. Good Autism Practice (GAP), 12(1), 34-41. Retrieved from http://docserver.ingentaconnect.com/deliver/connect/bild/14662973/v12n1/s5.pdf?expire $\mathrm{S}=1458573512 \& \mathrm{id}=86436442 \&$ titleid $=75007062 \&$ accname $=\mathrm{UCL}+\mathrm{LIBRARY} \& \mathrm{checksu}$ $\mathrm{m}=67 \mathrm{DD} 45 \mathrm{~F} 10 \mathrm{~B} 3 \mathrm{BC} 0 \mathrm{~F} 23 \mathrm{C} 260 \mathrm{~A} 42 \mathrm{~F} 5 \mathrm{~F} 40513$

Head, A. M., McGillivray, J. A., \& Stokes, M. A. (2014). Gender differences in emotionality and sociability in children with autism spectrum disorders. Molecular Autism, 5(19). Retrieved from http://www.biomedcentral.com/content/pdf/2040-2392-5-19.pdf

Hull, L., Petrides, K. V., Allison, C., Smith, P., Baron-Cohen, S., Lai, M.-C., \& Mandy, W. (2017). "Putting on My Best Normal": Social Camouflaging in Adults with Autism Spectrum Conditions. Journal of Autism and Developmental Disorders, 47(8). https://doi.org/10.1007/s10803-017-3166-5 
Hull, Laura, Mandy, W., Lai, M., Baron-Cohen, S., Allison, C., Smith, P., \& Petrides, K. V. (2018). Development and Validation of the Camouflaging Autistic Traits Questionnaire (CAT-Q). Journal of Autism and Developmental Disorders, 49(3), 819-833. https://doi.org/10.1007/s10803-018-3792-6

Hurley, R. S. E., Losh, M., Parlier, M., Reznick, J. S., \& Piven, J. (2007). The broad autism phenotype questionnaire. Journal of Autism and Developmental Disorders, 37(9), 16791690. https://doi.org/10.1007/s10803-006-0299-3

IBM Corp. (2015). IBM SPSS Statistics for Windows, Version 23.0. Armonk, NY: IBM Corp.

Kirkovski, M., Enticott, P. G., \& Fitzgerald, P. B. (2013). A review of the role of female gender in autism spectrum disorders. Journal of Autism and Developmental Disorders, 43(11), 2584-2603. https://doi.org/10.1007/s10803-013-1811-1

Kopp, S., \& Gillberg, C. (1992). Girls with social deficits and learning problems: Autism, atypical Asperger syndrome or a variant of these conditions. European Child \& Adolescent Psychiatry, 1(2), 89-99. https://doi.org/10.1007/BF02091791

Kreiser, N. L., \& White, S. W. (2014). ASD in females: are we overstating the gender difference in diagnosis? Clinical Child and Family Psychology Review, 17(1), 67-84. https://doi.org/10.1007/s10567-013-0148-9

Lai, M.-C., Lombardo, M. V., Ruigrok, A. N. V., Chakrabarti, B., Auyeung, B., Szatmari, P., ... Consortium, M. A. (2017). Quantifying and exploring camouflaging in men and women with autism. Autism, 21(6), 690-702. https://doi.org/10.1177/1362361316671012

Lai, M.-C., Lombardo, M. V, Auyeung, B., Chakrabarti, B., \& Baron-Cohen, S. (2015). 
Gender Differences in Camouflaging

Sex/gender differences and autism: setting the scene for future research. Journal of the American Academy of Child and Adolescent Psychiatry, 54(1), 11-24.

https://doi.org/10.1016/j.jaac.2014.10.003

Lai, M.-C., Lombardo, M. V, Chakrabarti, B., Ruigrok, A. N., Bullmore, E. T., Suckling, J., ... Baron-Cohen, S. (2018). Neural self-representation in autistic women and association with 'compensatory camouflaging.' Autism, 136236131880715. https://doi.org/10.1177/1362361318807159

Lai, M.-C., Lombardo, M. V, Pasco, G., Ruigrok, A. N. V, Wheelwright, S. J., Sadek, S. A., ... Baron-Cohen, S. (2011). A behavioral comparison of male and female adults with high functioning autism spectrum conditions. PloS One, 6(6), e20835. https://doi.org/10.1371/journal.pone.0020835

Lehnhardt, F.-G., Falter, C. M., Gawronski, A., Pfeiffer, K., Tepest, R., Franklin, J., \& Vogeley, K. (2015). Sex-related cognitive profile in Autism Spectrum Disorders diagnosed late in life: Implications for the Female Autistic Phenotype. Journal of Autism and Developmental Disorders, 46(1), 139-154. https://doi.org/10.1007/s10803-015$2558-7$

Lehnhardt, F.-G., Gawronski, A., Pfeiffer, K., Kockler, H., Schilbach, L., \& Vogeley, K. (2013). The investigation and differential diagnosis of Asperger syndrome in adults. Deutsches Ärzteblatt International, 110(45), 755-763. https://doi.org/10.3238/arztebl.2013.0755

Lenhard, W., \& Lenhard, A. (2016). Calculation of Effect Sizes. https://doi.org/10.13140/RG.2.1.3478.4245

Livingston, L. A., Colvert, E., Bolton, P., \& Happé, F. (2018). Good social skills despite poor theory of mind: exploring compensation in autism spectrum disorder. Journal of Child 
Psychology and Psychiatry. https://doi.org/10.1111/jcpp.12886

Livingston, L. A., \& Happé, F. (2017). Conceptualising compensation in neurodevelopmental disorders: Reflections from Autism Spectrum Disorder. Neuroscience and Biobehavioral Reviews. https://doi.org/10.1016/j.neubiorev.2017.06.005

MacPhee, D., \& Prendergast, S. (2019). Room for Improvement: Girls' and Boys' Home Environments are Still Gendered. Sex Roles, 80(5-6), 332-346. https://doi.org/10.1007/s11199-018-0936-2

Nishiyama, T., Suzuki, M., Adachi, K., Sumi, S., Okada, K., Kishino, H., ... Kanne, S. M. (2014). Comprehensive comparison of self-administered questionnaires for measuring quantitative autistic traits in adults. Journal of Autism and Developmental Disorders, 44(5), 993-1007. https://doi.org/10.1007/s10803-013-2020-7

Parish-Morris, J., Liberman, M. Y., Cieri, C., Herrington, J. D., Yerys, B. E., Bateman, L., ... Schultz, R. T. (2017). Linguistic camouflage in girls with autism spectrum disorder. Molecular Autism, 8(1), 48. https://doi.org/10.1186/s13229-017-0164-6

Prendergast, S., \& MacPhee, D. (2018). Family Resilience Amid Stigma and Discrimination: A Conceptual Model for Families Headed by Same-Sex Parents. Family Relations, 67(1), 26-40. https://doi.org/10.1111/fare.12296

R Core Team. (2013). R: A language and environment for statistical processing. Vienna, Austria: R Foundation for Statistical Computing. Retrieved from http://www.rproject.org/

Ratto, A. B., Kenworthy, L., Yerys, B. E., Bascom, J., Trubanova, A., White, S. W., ... Anthony, G. (2018). What About the Girls? Sex-Based Differences in Autistic Traits and Adaptive Skills. Journal of Autism and Developmental Disorders, 48(5), 1698- 
1711. https://doi.org/10.1007/s10803-017-3413-9

Robinson, E. B., Munir, K., Munafò, M. R., Hughes, M., McCormick, M. C., \& Koenen, K. C. (2011). Stability of autistic traits in the general population: further evidence for a continuum of impairment. Journal of the American Academy of Child and Adolescent Psychiatry, 50(4), 376-384. https://doi.org/10.1016/j.jaac.2011.01.005

Sasson, N. J., Lam, K. S. L., Childress, D., Parlier, M., Daniels, J. L., \& Piven, J. (2013). The Broad Autism Phenotype Questionnaire: Prevalence and diagnostic classification. Autism Research, 6(2), 134-143. https://doi.org/10.1002/aur.1272.The

Sedgewick, F., Hill, V., Yates, R., Pickering, L., \& Pellicano, E. (2015). Gender Differences in the Social Motivation and Friendship Experiences of Autistic and Non-autistic Adolescents. Journal of Autism and Developmental Disorders, 46(4), 1297-1306. https://doi.org/10.1007/s10803-015-2669-1

Shattuck, P. T., Durkin, M., Maenner, M., Newschaffer, C., Mandell, D. S., Wiggins, L., ... Cuniff, C. (2009). Timing of identification among children with an autism spectrum disorder: findings from a population-based surveillance study. Journal of the American Academy of Child and Adolescent Psychiatry, 48(5), 474-483. https://doi.org/10.1097/CHI.0b013e31819b3848

Tierney, S., Burns, J., \& Kilbey, E. (2016). Looking behind the mask: Social coping strategies of girls on the autistic spectrum. Research in Autism Spectrum Disorders, 23, 73-83. https://doi.org/10.1016/j.rasd.2015.11.013 


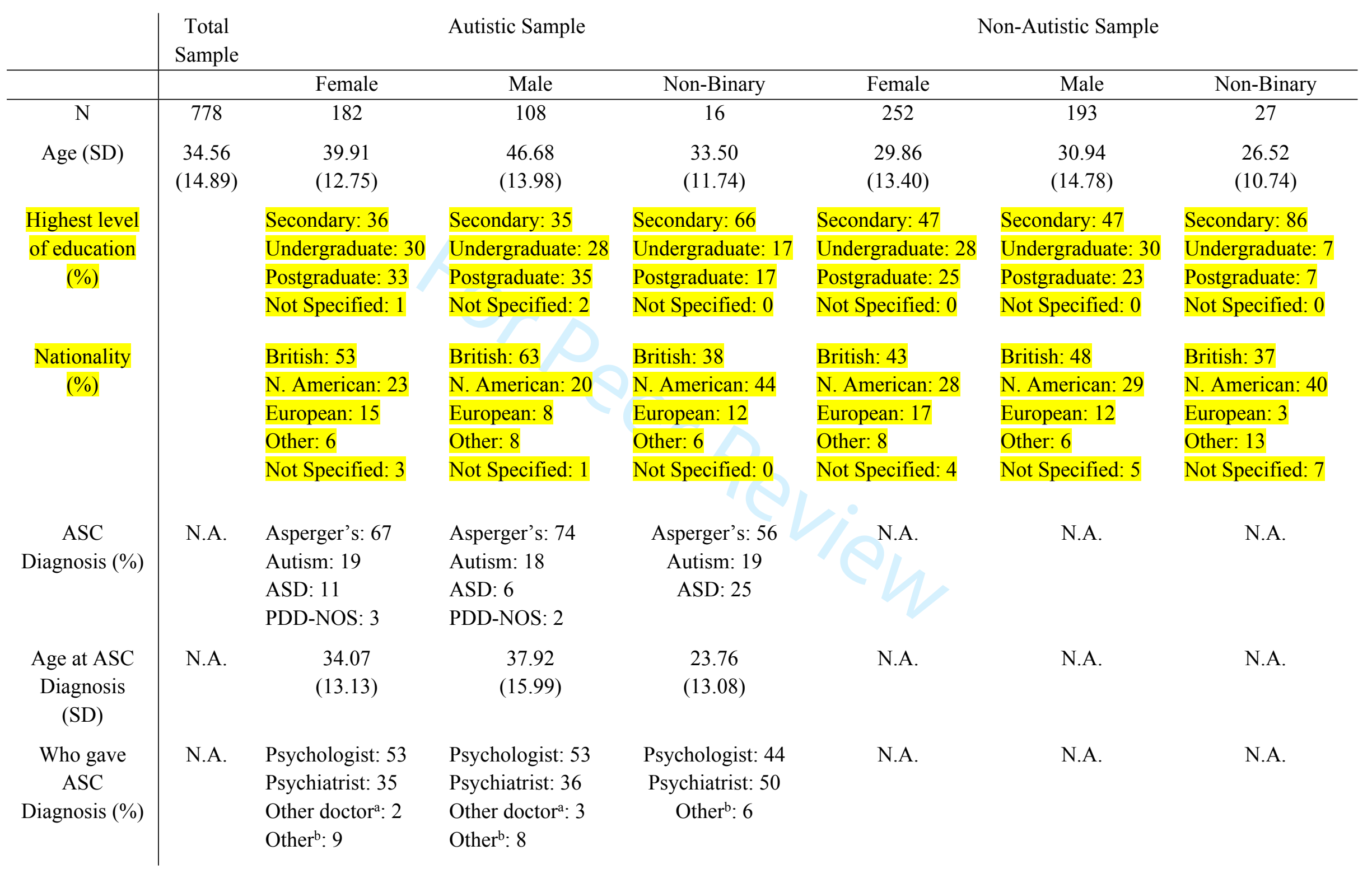




\begin{tabular}{c|ccccccc} 
CAT-Q Total & 104.16 & 124.35 & 109.64 & 122.00 & 90.87 & 96.89 & $(24.22)$ \\
(SD) & $(28.54)$ & $(23.27)$ & $(26.50)$ & $(17.12)$ & $(27.67)$ & $(27.20)$ \\
CAT-Q Comp & 33.37 & 41.85 & 36.81 & 43.50 & 27.18 & 30.06 & $(10.92)$ \\
(SD) & $(12.73)$ & $(11.11)$ & $(12.14)$ & $(9.89)$ & $(11.50)$ & 35.48 \\
CAT-Q Mask & 35.80 & 37.87 & 32.90 & 36.06 & 34.69 & 36.34 & $(11.32)$ \\
(SD) & $(9.51)$ & $(10.54)$ & $(10.57)$ & $(8.78)$ & $(9.05)$ & $38.13)$ & $(7.61)$ \\
CAT-Q Assim & 34.94 & 44.63 & 39.93 & 39.88 & 29.00 & 30.48 & $(10.33)$ \\
(SD) & $(12.20)$ & $(7.82)$ & $(11.26)$ & $(6.43)$ & $(11.73)$ & 35.26 \\
BAPQ Total & 3.63 & 4.37 & 4.17 & 4.30 & $(12.11)$ \\
(SD) & $(0.91)$ & $(0.63)$ & $(0.84)$ & $(0.50)$ & $(0.73)$ & 3.24 & $(0.72)$
\end{tabular}

Note. ASC = Autism Spectrum Condition; PDD-NOS = Pervasive Developmental Disorder, Not Otherwise Specified; CAT-Q = Camouflaging Autistic Traits Questionnaire; CAT-Q Comp = Compensation subscale; CAT-Q Mask = Masking subscale; CAT-Q Assim = Assimilation subscale; BAPQ = Broader Autism Phenotype Questionnaire. ${ }^{a}$ Including family doctor/general practitioner, paediatrician, and multi-disciplinary team. ${ }^{\mathrm{b}}$ Including speech and language therapist, school, and nurse practitioner. 
Figure 1. Distribution of Total CAT-Q scores

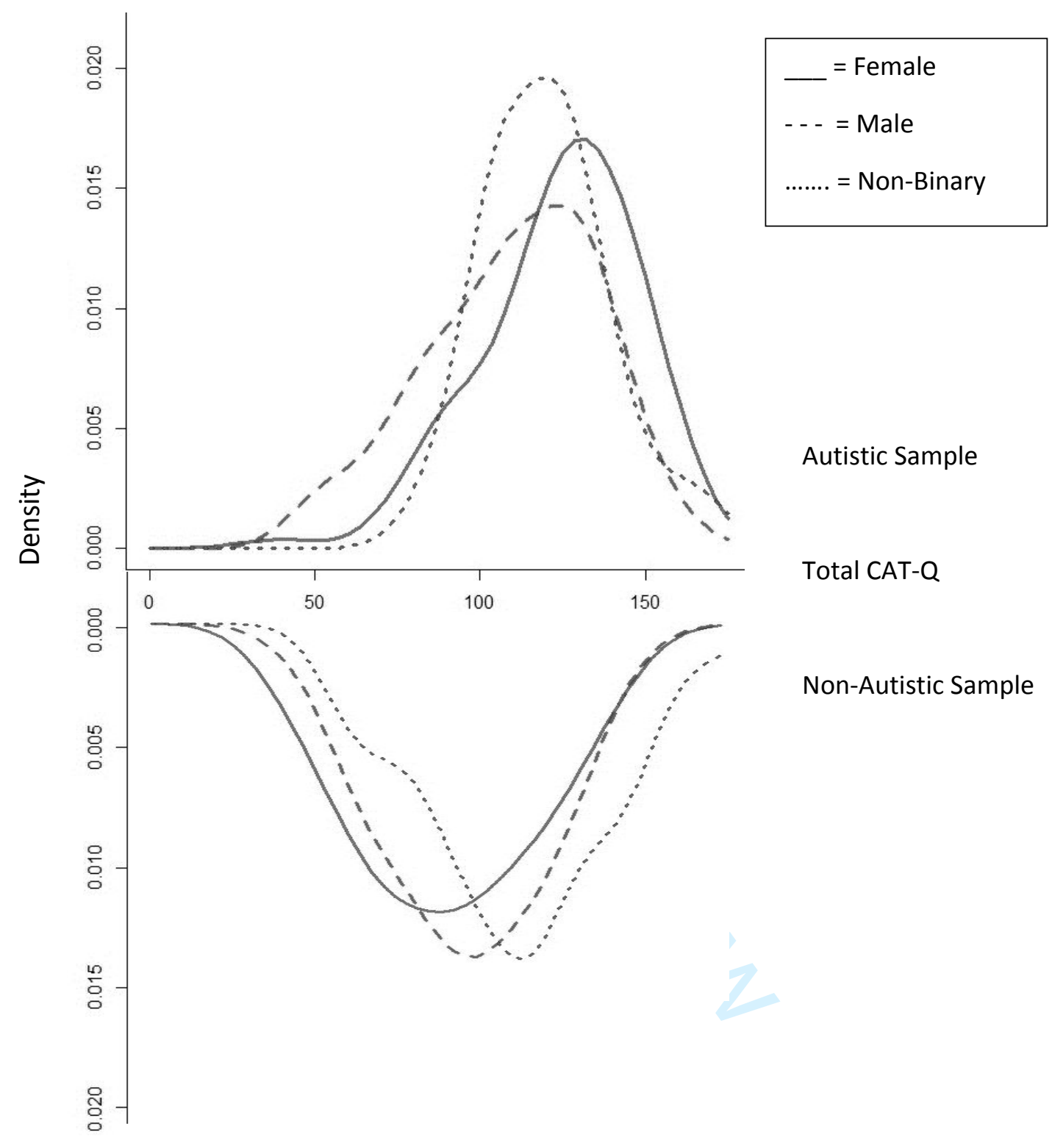


Figure 2. Mean Total CAT-Q (a), Compensation (b), Masking (c), and Assimilation (d) scores by Group and Gender, controlling for age
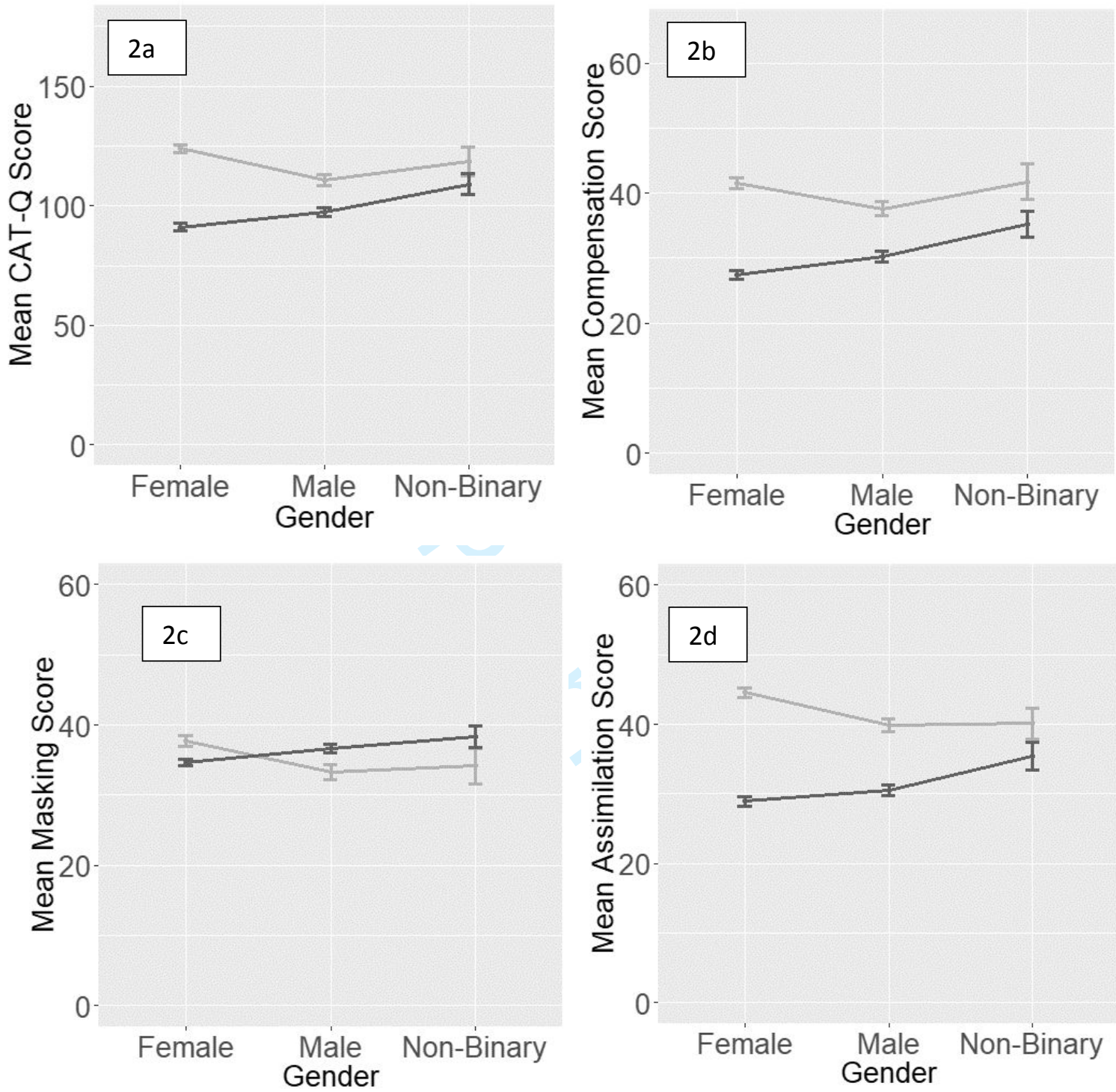

Diagnostic Group

- Autistic

- Non-Autistic 
Figure 3. Mean Total CAT-Q (a), Compensation (b), Masking (c), and Assimilation (d) scores by Group and Gender, controlling for age and autistic-like traits
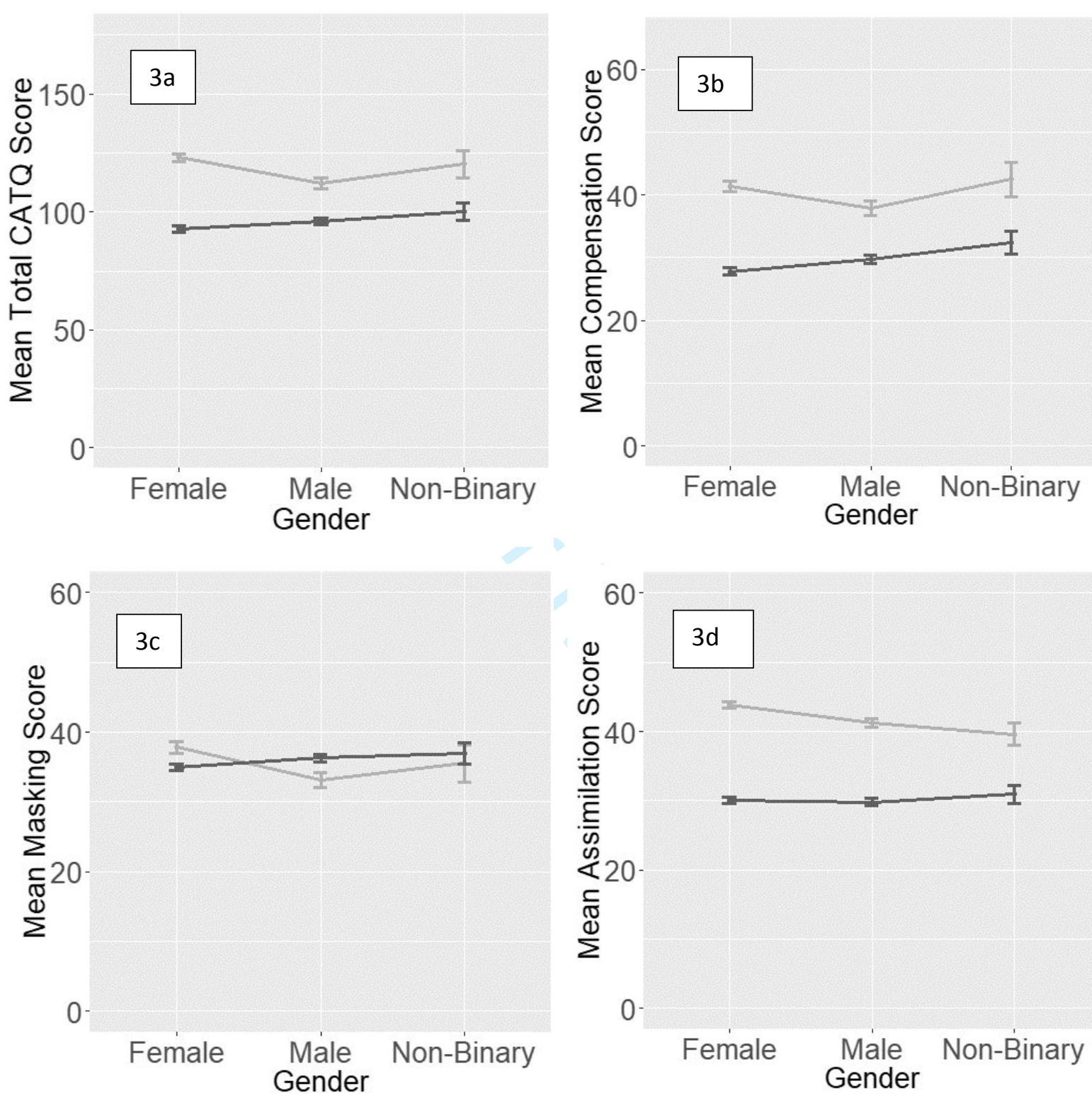

\section{Diagnostic Group}

- Autistic

- Non-Autistic 
Supplementary Figures 1a-c. Distribution of Compensation (SF1a), Masking (SF1b) and Assimilation (SF1c) subscale scores in Autistic (above x-axis) and Non-Autistic (below x-axis) samples

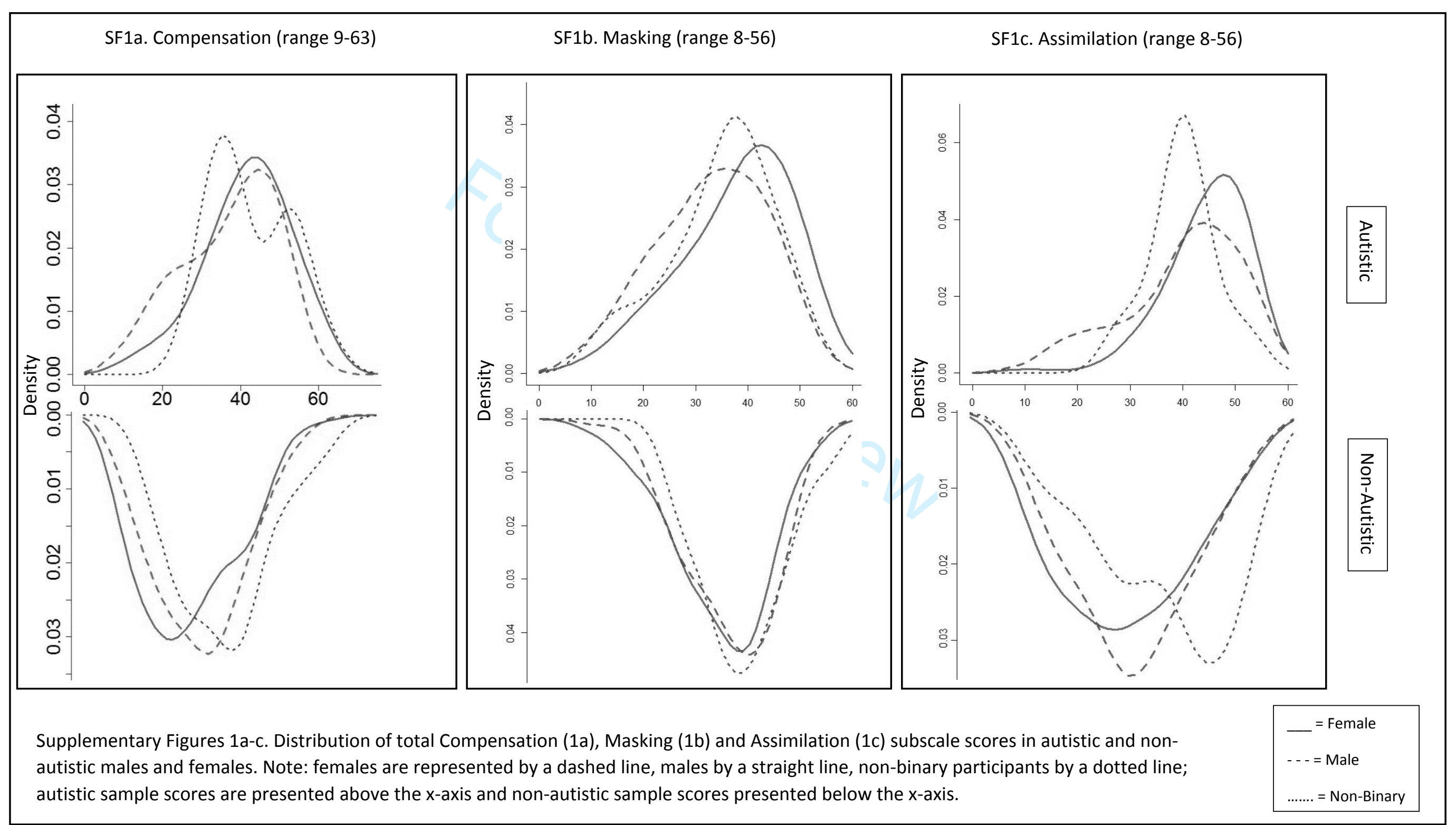

http://mc.manuscriptcentral.com/autism 